OPEN ACCESS

Edited by:

David M. Labiner,

University of Arizona, United States

Reviewed by:

Peter W. Kaplan,

SOURCE, Johns Hopkins University,

United States

Elena Gardella

University of Southern

Denmark, Denmark

*Correspondence:

Anthony L. Fine

fine.anthony@mayo.edu

Specialty section

This article was submitted to

Epilepsy,

a section of the journal

Frontiers in Neurology

Received: 17 September 2021

Accepted: 28 October 2021

Published: 29 November 2021

Citation:

Fine AL, Liebo G, Gavrilova RH and Britton JW (2021) Seizure Semiology, EEG, and Imaging Findings in Epilepsy Secondary to Mitochondrial Disease.

Front. Neurol. 12:779052.

doi: 10.3389/fneur.2021.779052

\section{Seizure Semiology, EEG, and Imaging Findings in Epilepsy Secondary to Mitochondrial Disease}

\author{
Anthony L. Fine ${ }^{1 *}$, Greta Liebo ${ }^{2}$, Ralitza H. Gavrilova ${ }^{1,3}$ and Jeffrey W. Britton ${ }^{1}$ \\ ${ }^{1}$ Department of Neurology, Mayo Clinic, Rochester, MN, United States, ${ }^{2}$ Department of Radiology, Mayo Clinic, Rochester, \\ MN, United States, ${ }^{3}$ Department of Clinical Genomics, Mayo Clinic, Rochester, MN, United States
}

Background: Identification of an underlying mitochondrial disorder can be challenging due to the significant phenotypic variability between and within specific disorders. Epilepsy can be a presenting symptom with several mitochondrial disorders. In this study, we evaluated clinical, electrophysiologic, and imaging features in patients with epilepsy and mitochondrial disorders to identify common features, which could aid in earlier identification of a mitochondrial etiology.

Methods: This is a retrospective case series from January 2011 to December 2019 at a tertiary referral center of patients with epilepsy and a genetically confirmed diagnosis of a mitochondrial disorder. A total of 164 patients were reviewed with 20 patients fulfilling inclusion criteria.

Results: A total of 20 patients (14 females, 6 males) aged 0.5-61 years with epilepsy and genetically confirmed mitochondrial disorders were identified. Status epilepticus occurred in 15 patients, with focal status epilepticus in 13 patients, including 9 patients with visual features. Abnormalities over the posterior cerebral regions were seen in $66 \%$ of ictal recordings and $44 \%$ of imaging studies. All the patients were on nutraceutical supplementation with no significant change in disease progression seen. At last follow-up, eight patients were deceased and the remainder had moderate-to-severe disability.

Discussion: In this series of patients with epilepsy and mitochondrial disorders, we found increased propensity for seizures arising from the posterior cerebral regions. Over time, electroencephalogram (EEG) and imaging abnormalities increasingly occurred over the posterior cerebral regions. Focal seizures and focal status epilepticus with visual symptoms were common. Additional study is needed on nutraceutical supplementation in mitochondrial disorders.

Keywords: EEG, mitochondria, genetic, neuroimaging, epilepsy

\section{INTRODUCTION}

Disorders of mitochondrial function and metabolism result in energy failure producing varying severity of symptoms (1-3). The central nervous system is particularly susceptible to mitochondrial defects and neurologic manifestations are frequently seen in mitochondrial disorders $(4,5)$.

Seizures are particularly common in mitochondrial disorders such as polymerase gamma (POLG) mutations, mitochondrial encephalomyopathy, lactic acidosis, and stroke-like episodes 
(MELAS), and myoclonic epilepsy with ragged-red fibers (MERRF) (6-9). Published information on specific seizure semiology and electroencephalogram (EEG) findings in mitochondrial disorders is limited compared to what is known about other genetic and metabolic epilepsies, likely owing to the significant variability between and within specific mitochondrial disorders (10). While seizures are not unique to a mitochondrial etiology (11-13), it has been previously noted that EEG abnormalities in these disorders tend to have a posterior cerebral predilection (14-16). Within the central nervous system, mitochondrial dysfunction frequently results in structural injury, which can manifest as characteristic neuroimaging abnormalities $(17,18)$. The time to diagnosis can be variable and in some cases may take years when presentations are atypical or when symptoms and diagnostic studies are non-specific. Treatment of these disorders is limited and mainly comprised of utilization of nutritional therapies aimed to increase the metabolic substrates used in mitochondrial processes (19). These nutraceutical therapies, sometimes referred to as the "mitochondrial cocktail," are unproven at present with limited evidence-based support of their use.

The main objective of this study was to evaluate the EEG, clinical, and radiologic progression of disease in patients with epilepsy due to mitochondrial cytopathies in order to identify features, which may help the clinician more readily diagnose these disorders. A secondary objective was to study nutraceutical therapy use in relationship to disease progression.

\section{MATERIALS AND METHODS}

This retrospective study was performed using the Mayo Clinic Mitochondrial Disease Database from January 2011 to December 2019. Records were reviewed for diagnosis, history of epilepsy, laboratory investigations, and available raw EEG and MRI studies. Patients were classified based on previously published diagnostic criteria as having a definite, probable, or possible mitochondrial disorder (20-23).

Patients with a definite, genetically confirmed diagnosis of mitochondrial disorder were included. Patients without confirmatory testing or where there was diagnostic ambiguity were excluded. Additional inclusion criteria included history of seizures and EEG and MRI studies performed at our institution. Patients were excluded if any of the inclusion criteria were missing or if medical records were limited.

Information extracted from the medical record included seizure history and semiology, anti-seizure therapies and side effects, laboratory investigations, including genetic testing and tissue biopsy results, EEG and neuroimaging findings, use of nutraceuticals/supplements, and disposition at last follow-up. Seizure semiology was classified based upon the most recent International League Against Epilepsy (ILAE) classification schema $(24,25)$.

Electroencephalogram studies were graded based on the degree and location of abnormalities and rated as either normal or demonstrating mild, moderate, or severe abnormalities using the Mayo Clinic EEG classification system, which assigns a dysrhythmia score of 1 through 3 ( $1=$ mild, $2=$ moderate, and $3=$ severe). This included pediatric EEG recordings, which were reviewed and interpreted with attention to ageexpected findings in children. "Mild" was defined as background slowing of 7-8 Hertz $(\mathrm{Hz})$ or intermittent slowing in the theta range $(5-7 \mathrm{~Hz})$; "moderate" if there was a mixture of frequent delta-theta slowing $(<4 \mathrm{~Hz}$ up to $7 \mathrm{~Hz})$ or if there was a mild-to-moderate asymmetry of the background ( $<50 \%$ difference in amplitude between homologous regions); and "severe" if any of the following were present: focal or diffuse delta slowing $(<4 \mathrm{~Hz})$, severe background asymmetry ( $>75 \%$ difference in amplitude between homologous regions), epileptiform abnormalities [spikes, spike and wave, and sharp wave discharges, lateralized periodic discharges (LPDs) or generalized periodic discharges (GPDs), and periodic sharp wave complexes], or recorded seizures or status epilepticus. The locations of EEG abnormalities were noted and categorized as focal, multifocal (focal abnormalities in more than two regions), or generalized.

Magnetic resonance imaging studies were reviewed by one study author (GL) and abnormalities noted based on location, imaging sequence [T2/fluid-attenuated inversion recovery (FLAIR) and diffusion-weighted imaging (DWI)], abnormal contrast enhancement, and presence or absence of cortical laminar necrosis, atrophy, or encephalomalacia.

Electroencephalogram and MRI results were evaluated for anatomic concordance, defined as colocalization on studies, comparing studies obtained within 30 days of each other. Serial EEG and MRI studies were also evaluated for disease progression, defined as the presence of new abnormalities compared to previous studies.

Functional status was assigned based on initial and last documented encounters, using the modified Rankin scale (mRS), with a score of 0 (no symptoms), 1 (no significant disability), 2 (slight disability), 3 (moderate disability), 4 (moderate-to-severe disability), 5 (severe disability), or 6 (death). Patient disposition was determined at last known follow-up based on notes or communications contained in the medical record.

This study was approved by the Mayo Clinic Foundation Institutional Review Board.

\section{RESULTS}

A total of 164 unique patient records were reviewed with 144 patients excluded for the following reasons: limited/missing clinical information $(n=22)$, absence of seizures $(n=113)$, or diagnosis of a probable or possible mitochondrial disorder $(n=9)$.

Diagnoses in excluded patients included MELAS (m.3243A $>$ G variant) $(n=28)$, chronic progressive external ophthalmoplegia (CPEO)/Kearns-Sayre syndrome (KSS) $(n=14)$, Leber hereditary optic neuropathy (LHON) $(n=5)$, neuropathy, ataxia, and retinitis pigmentosa (NARP) $(n=2)$, and Leigh syndrome $(n=2)$. In an additional 34 patients without seizure, the diagnosis was based on history and laboratory findings without genetic confirmation. 
TABLE 1 | Seizure characteristics and functional status of patient.

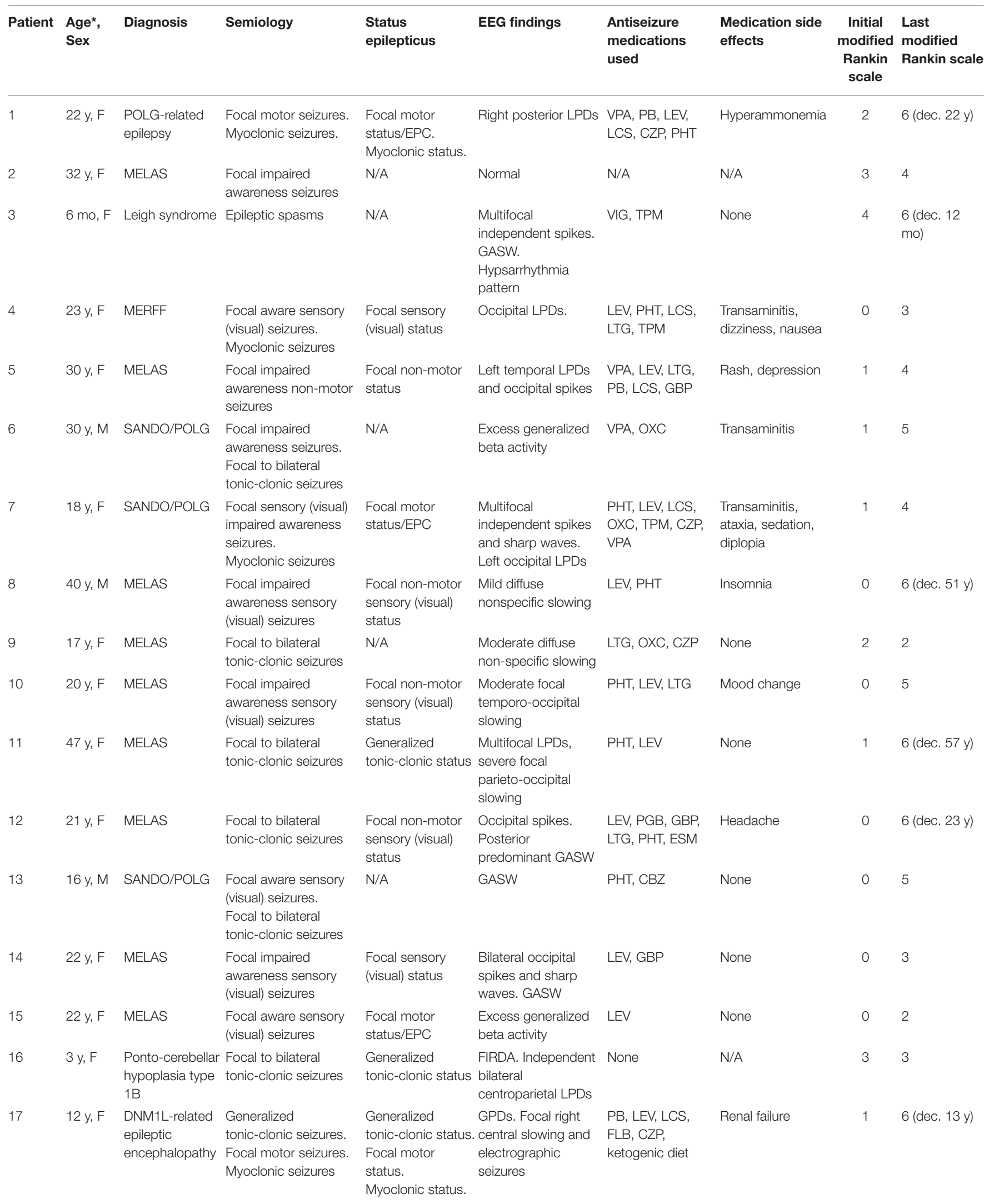




\begin{tabular}{|c|c|c|c|c|c|c|c|c|c|}
\hline Patient & $\begin{array}{l}\text { Age* }^{*} \\
\text { Sex }\end{array}$ & Diagnosis & Semiology & $\begin{array}{l}\text { Status } \\
\text { epilepticus }\end{array}$ & EEG findings & $\begin{array}{l}\text { Antiseizure } \\
\text { medications } \\
\text { used }\end{array}$ & $\begin{array}{l}\text { Medication side } \\
\text { effects }\end{array}$ & $\begin{array}{l}\text { Initial } \\
\text { modified } \\
\text { Rankin } \\
\text { scale }\end{array}$ & $\begin{array}{l}\text { Last } \\
\text { modified } \\
\text { Rankin scale }\end{array}$ \\
\hline 18 & 13 y, M & MELAS & Focal motor seizures & $\begin{array}{l}\text { Generalized } \\
\text { tonic-clonic status. } \\
\text { Focal sensory } \\
\text { (visual) status }\end{array}$ & $\begin{array}{l}\text { Right occipital LPDs. } \\
\text { Posterior } \\
\text { predominant } \\
\text { multifocal spikes }\end{array}$ & $\begin{array}{l}\text { LEV, PHT, LTG, } \\
\text { CZP }\end{array}$ & None & 0 & 6 (dec. 29 y) \\
\hline 19 & $12 \mathrm{y}, \mathrm{M}$ & MELAS & $\begin{array}{l}\text { Focal aware sensory } \\
\text { (visual) seizures }\end{array}$ & $\begin{array}{l}\text { Focal sensory } \\
\text { (visual) status }\end{array}$ & $\begin{array}{l}\text { Bi-parieto-occipital } \\
\text { LPDs }\end{array}$ & $\begin{array}{l}\text { VPA, LEV, LCS, } \\
\text { LTG, PHT }\end{array}$ & $\begin{array}{l}\text { Stevens-Johnson } \\
\text { syndrome }\end{array}$ & 0 & 6 (dec. 25 y) \\
\hline 20 & $61 \mathrm{y}, \mathrm{M}$ & MELAS & $\begin{array}{l}\text { Focal impaired } \\
\text { awareness seizures }\end{array}$ & $\begin{array}{l}\text { Focal non-motor } \\
\text { status }\end{array}$ & $\begin{array}{l}\text { Independent } \\
\text { bitemporal LPDs }\end{array}$ & LEV & None & 1 & 5 \\
\hline
\end{tabular}

\section{${ }^{*}$ Age at presentation.}

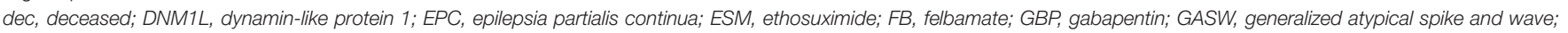

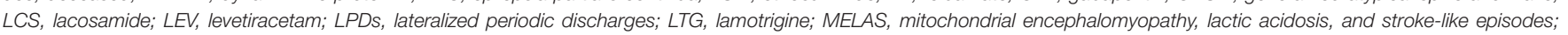

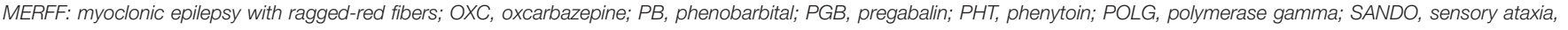
neuropathy, dysarthria, and ophthalmoparesis; TPM, topiramate; VIG, vigabatrin; VPA, valproic acid.

Table 1 shows details on the 20 patients that fulfilled inclusion criteria. The mean age at initial evaluation was 23 years (median 21.5 years, SD 14.3 years) with 6 males and 14 females. Two kindred pairs were included (one sibling pair and one parentchild pair). The most common diagnoses were MELAS $(n=12)$ and POLG1 $(n=3)$. Evaluation of epilepsy was the most common initial visit indication $(n=14)$, followed by genetics evaluation $(n=2)$, developmental delay $(n=2)$, and headache evaluation $(n=2)$.

\section{Metabolic and Genetic Testing}

All the patients underwent biochemical and metabolic analyses including some combination of blood, urine, and cerebrospinal fluid (CSF) testing (Table 2). Studies commonly performed included urine organic acids, serum amino acids, serum lactate and pyruvate, comprehensive fatty acid profile, carnitine, and acylcarnitine profile. The most commonly seen abnormalities on blood testing were an elevated lactate $(n=17)$, pyruvate $(n=10)$, and alanine $(n=7)$. Spinal fluid analysis was performed in seven patients, with elevated lactate and pyruvate seen in two patients and elevated CSF protein $(>35 \mathrm{mg} / \mathrm{dl})$ in six patients. Muscle biopsy was performed in five patients and fibroblast culture and analysis was performed in three patients (Table 2).

Genetic testing performed included point mutation analysis $(n=9)$, next-generation sequencing $(n=8)$, and whole-exome sequencing $(n=3)$. Heteroplasmy analysis was available in 11 patients with values ranging from 6 to 97\% (Table 2). In the patient with $6 \%$ heteroplasmy in blood, the clinical phenotype was felt to be consistent with MELAS, with m.3243A $>$ G variant, and muscle biopsy was recommended but declined by the patient.

\section{Seizure Semiology}

The most common seizure semiology was focal seizures in 19 patients, with the exception of the patient with epileptic spasms. Focal semiologies included focal impaired awareness $(n=8)$, focal aware sensory seizures $(n=4)$, focal to bilateral tonic-clonic seizures $(n=6)$, and focal motor seizures $(n=3)$. This includes two patients who had both focal and focal to bilateral tonicclonic seizures and one patient with a history of both focal motor seizures and generalized tonic-clonic seizures (although focal to bilateral tonic-clonic seizures cannot be excluded). Ictal visual sensory symptoms were experienced by eight patients (40\%), with four patients who had retained awareness and four patients who experienced loss of awareness during seizures. Visual features described included ictal blindness, visual distortions, scintillating scotomas, hallucinations, palinopsia, and photopsia.

A total of 15 patients (75\%) had a history of status epilepticus including 4 patients as their initial presenting symptom. Focal status epilepticus occurred in 13 patients, with focal motor status/epilepsia partialis continua occurring in 4 patients and focal non-convulsive status seen in 9 patients, with 7 patients experiencing focal sensory status with visual symptoms. Four patients had generalized tonic-clonic status epilepticus including two patients who experienced both focal and generalized status epilepticus on different occasions. Two patients experienced super-refractory myoclonic status epilepticus, with ultimate withdrawal of medical support resulting in death.

\section{Electroencephalogram Findings}

A total of 64 EEG recordings were performed: 37 outpatient routine recordings and 27 inpatient continuous recordings. A total of 14 patients had more than one EEG recording performed (range 2-11, mode 2). Eight patients had both outpatient routine and inpatient continuous recordings performed. In the remaining patients, an additional eight patients had only outpatient routine recordings and four patients had only prolonged hospital studies performed.

Outpatient EEG studies were classified as normal in two patients, mildly abnormal in three patients (excess fast activity or excessive drowsiness), moderately abnormal in three patients (focal delta-theta slowing), and severely abnormal in eight patients [generalized atypical spike and wave (four patients), focal and multifocal discharges (three patients), and lateralized periodic discharges (one patient)]. Two of the four patients with 
TABLE 2 | Phenotypes, genetic, and biochemical testing of patient.

\begin{tabular}{|c|c|c|c|c|c|c|c|}
\hline Patient & Age, sex & Phenotype & Diagnosis & Gene, variant & $\begin{array}{l}\text { Heteroplasmy } \\
\text { (source) }\end{array}$ & $\begin{array}{l}\text { Biochemical } \\
\text { testing }\end{array}$ & $\begin{array}{l}\text { Muscle biopsy } \\
\text { /fibroblasts }\end{array}$ \\
\hline 1 & $22 \mathrm{y}, \mathrm{F}$ & Developmental delay, epilepsy & $\begin{array}{l}\text { POLG-related } \\
\text { epilepsy }\end{array}$ & $\begin{array}{l}\text { POLG1, c.1491G>C, } \\
\text { POLG1, c. } 2243 G>C\end{array}$ & N/A & $\begin{array}{l}\text { Elevated serum } \\
\text { lactate }\end{array}$ & $\begin{array}{l}\text { Cytochrome } c \\
\text { oxidase negative } \\
\text { fibers }\end{array}$ \\
\hline 2 & $32 \mathrm{y}, \mathrm{F}$ & $\begin{array}{l}\text { Short stature, thin body } \\
\text { habitus, hearing loss, epilepsy, } \\
\text { diabetes, cardiac arrhythmias, } \\
\text { ataxia }\end{array}$ & MELAS & MTTL1, m.3243A>G & Unknown & $\begin{array}{l}\text { Elevated serum } \\
\text { lactate, pyruvate, } \\
\text { and alanine }\end{array}$ & $\mathrm{N} / \mathrm{A}$ \\
\hline 3 & $6 \mathrm{mo}, \mathrm{F}$ & $\begin{array}{l}\text { Developmental delay, failure to } \\
\text { thrive, infantile spasms, } \\
\text { hypotonia, Wolf-Parkinson } \\
\text { White syndrome }\end{array}$ & $\begin{array}{l}\text { Leigh } \\
\text { syndrome }\end{array}$ & ND5, m.13513G>A & $72.6 \%$ (blood) & $\begin{array}{l}\text { Elevated serum } \\
\text { lactate and } \\
\text { pyruvate }\end{array}$ & $\mathrm{N} / \mathrm{A}$ \\
\hline 4 & 23 y, F & $\begin{array}{l}\text { Short stature, thin body } \\
\text { habitus, epilepsy, intellectual } \\
\text { disability }\end{array}$ & MERFF & MTTL1, m.3256C>T & $40.7 \%$ (blood) & $\begin{array}{l}\text { Elevated serum } \\
\text { lactate }\end{array}$ & $\begin{array}{l}\text { Fibroblasts: } \\
\text { normal. }\end{array}$ \\
\hline 6 & $30 \mathrm{y}, \mathrm{M}$ & $\begin{array}{l}\text { Progressive external } \\
\text { ophthalmoplegia, epilepsy, } \\
\text { developmental } \\
\text { delay/intellectual disability, } \\
\text { mood disorder, peripheral } \\
\text { neuropathy, ataxia }\end{array}$ & SANDO & $\begin{array}{l}\text { POLG1, c. } 1491 G>C \\
\text { POLG1, c. } 2243 G>C\end{array}$ & $N / A$ & Normal & $\begin{array}{l}\text { Increased } \\
\text { sarcolemmal } \\
\text { oxidative reactivity }\end{array}$ \\
\hline 7 & 18 y, F & $\begin{array}{l}\text { Progressive external } \\
\text { ophthalmoplegia, epilepsy, } \\
\text { mood disorder, peripheral } \\
\text { neuropathy, ataxia }\end{array}$ & SANDO & $\begin{array}{l}\text { POLG1, c. } 1491 G>C \\
\text { POLG1, c. } 2243 G>C\end{array}$ & N/A & Normal & $\mathrm{N} / \mathrm{A}$ \\
\hline 8 & $40 \mathrm{y}, \mathrm{M}$ & $\begin{array}{l}\text { Short stature, hearing loss, } \\
\text { diabetes, cardiomyopathy, } \\
\text { stroke-like episodes, epilepsy }\end{array}$ & MELAS & MTTL1, m.3243A<G & Unknown & $\begin{array}{l}\text { Elevated serum } \\
\text { lactate and } \\
\text { pyruvate }\end{array}$ & $\mathrm{N} / \mathrm{A}$ \\
\hline 11 & 47 y, F & $\begin{array}{l}\text { Stroke-like episodes, hearing } \\
\text { loss, diabetes, short stature, } \\
\text { epilepsy }\end{array}$ & MELAS & MTTL1, m.3243A>G & 97\% (blood) & $\begin{array}{l}\text { Elevated serum } \\
\text { lactate }\end{array}$ & $\begin{array}{l}\text { Focal } \\
\text { subsarcolemmal } \\
\text { fuchsinophilic } \\
\text { material on } \\
\text { trichrome stain }\end{array}$ \\
\hline 12 & $21 \mathrm{y}, \mathrm{F}$ & $\begin{array}{l}\text { Epilepsy, peripheral } \\
\text { neuropathy, myopathy, } \\
\text { stroke-like episodes }\end{array}$ & MELAS & MTTL1, m.3243 A>G & $\begin{array}{l}89.6 \% \\
\text { (muscle) }\end{array}$ & $\begin{array}{l}\text { Elevated serum } \\
\text { lactate and } \\
\text { pyruvate }\end{array}$ & N/A \\
\hline 13 & $16 \mathrm{y}, \mathrm{M}$ & $\begin{array}{l}\text { Migraines, peripheral } \\
\text { neuropathy, ataxia, dysarthria, } \\
\text { progressive external } \\
\text { opthalmoplegia }\end{array}$ & SANDO & POLG1, c.1399G >A & N/A & Normal & $\mathrm{N} / \mathrm{A}$ \\
\hline 14 & $22 \mathrm{y}, \mathrm{F}$ & $\begin{array}{l}\text { Stroke-like episodes, epilepsy, } \\
\text { ataxia, diabetes, hearing loss, } \\
\text { cardiomyopathy }\end{array}$ & MELAS & MTTL1, m.3243A>G & Unknown & $\begin{array}{l}\text { Elevated serum } \\
\text { lactate }\end{array}$ & $\mathrm{N} / \mathrm{A}$ \\
\hline 15 & $22 y, F$ & $\begin{array}{l}\text { Short stature, thin body } \\
\text { habitus, progressive external } \\
\text { opthalmoplegia, ataxia, } \\
\text { myopathy, cardiomyopathy, } \\
\text { myoclonus, epilepsy }\end{array}$ & MELAS & MTTL1, m.3251A>G & 92\% (muscle) & $\begin{array}{l}\text { Elevated serum } \\
\text { lactate, pyruvate, } \\
\text { and alanine }\end{array}$ & $\begin{array}{l}\text { Ragged red fibers } \\
\text { with cytochrome c } \\
\text { oxidase activity }\end{array}$ \\
\hline
\end{tabular}


TABLE 2 | Continued

\begin{tabular}{|c|c|c|c|c|c|c|c|}
\hline Patient & Age, sex & Phenotype & Diagnosis & Gene, variant & $\begin{array}{l}\text { Heteroplasmy } \\
\text { (source) }\end{array}$ & $\begin{array}{l}\text { Biochemical } \\
\text { testing }\end{array}$ & $\begin{array}{l}\text { Muscle biopsy } \\
\text { /fibroblasts }\end{array}$ \\
\hline 16 & $3 y, F$ & $\begin{array}{l}\text { Global developmental delay, } \\
\text { hypotonia, spasticity, epilepsy, } \\
\text { ataxia }\end{array}$ & $\begin{array}{l}\text { Ponto- } \\
\text { cerebellar } \\
\text { hypoplasia } \\
\text { type 1B }\end{array}$ & $\begin{array}{l}\text { EXOSC3, c.395A>C } \\
\text { (homozygous) }\end{array}$ & $\mathrm{N} / \mathrm{A}$ & $\begin{array}{l}\text { Elevated serum } \\
\text { lactate }\end{array}$ & $\begin{array}{l}\text { Fibroblasts: } \\
\text { abnormal } \\
\text { mitochondrial } \\
\text { cristae } \\
\text { arrangements }\end{array}$ \\
\hline 18 & $13 y, M$ & $\begin{array}{l}\text { Stroke-like episodes, epilepsy, } \\
\text { headaches, hearing loss }\end{array}$ & MELAS & MTTL1, m.3243A>G & 70\% (muscle) & $\begin{array}{l}\text { Elevated serum } \\
\text { lactate and } \\
\text { pyruvate }\end{array}$ & N/A \\
\hline 19 & $12 \mathrm{y}, \mathrm{M}$ & $\begin{array}{l}\text { Stroke-like episodes, epilepsy, } \\
\text { headaches, mood disorder }\end{array}$ & MELAS & MTTL1, m.3243A>G & $40 \%$ (blood) & $\begin{array}{l}\text { Elevated serum } \\
\text { lactate and alanine }\end{array}$ & $\begin{array}{l}\text { Ragged blue } \\
\text { fibers, increased } \\
\text { succinate } \\
\text { dehydrogenase } \\
\text { enzyme reactivity }\end{array}$ \\
\hline 20 & 61 y, F & $\begin{array}{l}\text { Ataxia, hearing loss, diabetes, } \\
\text { stroke-like episodes, migraines }\end{array}$ & MELAS & $\mathrm{MTTL1}$, c.3243A>G & 6\% (blood) & $\begin{array}{l}\text { Elevated serum } \\
\text { and CSF lactate, } \\
\text { pyruvate, and } \\
\text { alanine }\end{array}$ & N/A \\
\hline
\end{tabular}

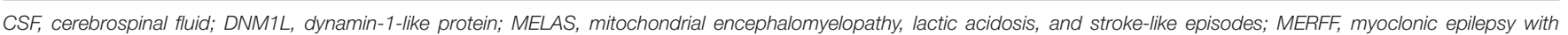
ragged-red fibers; POLG, polymerase gamma; SANDO, sensory ataxia, neuropathy, dysarthria, and ophthalmoparesis.

generalized atypical spike and wave activity had additional focal epileptiform findings and ictal EEG recordings contained focal onset seizures.

Prolonged inpatient recordings were performed in 12 patients (60\%). All prolonged recordings were classified as severely abnormal. Epileptiform abnormalities were focal in five patients, multifocal in three patients, generalized in one patient, and mixed (focal/multifocal and generalized) in three patients. Findings were notable for a predominance of discharges over the posterior/occipital head regions in eight patients (66\%) over 13 different studies in patients with diagnoses of MELAS $(n=6)$, POLG1 $(n=1)$, and MERRF $(n=1)$. In these patients, five patients experienced visual symptoms during seizures.

In the patients with normal or mildly abnormal outpatient EEG studies, four of the five patients continued to have normal or mildly abnormal outpatient EEG studies over time. Three patients with mild-to-moderately abnormal outpatient EEG studies had a clinical history of status epilepticus. Five patients with severely abnormal outpatient EEG recordings had a history of status epilepticus.

\section{Imaging Findings}

A total of 77 total MRI studies were performed. T2 hyperintensities were seen in 18 patients (90\%) and abnormal diffusion restriction was present in 8 patients (40\%). Imaging abnormalities were most commonly seen in the posterior head regions (Figure 1). Additional findings included cortical laminar necrosis $(n=3,15 \%)$, global cerebral atrophy $(n=$ $16,80 \%)$, dysmyelination $(n=5,25 \%)$, and abnormal contrast enhancement $(n=3,15 \%)$.

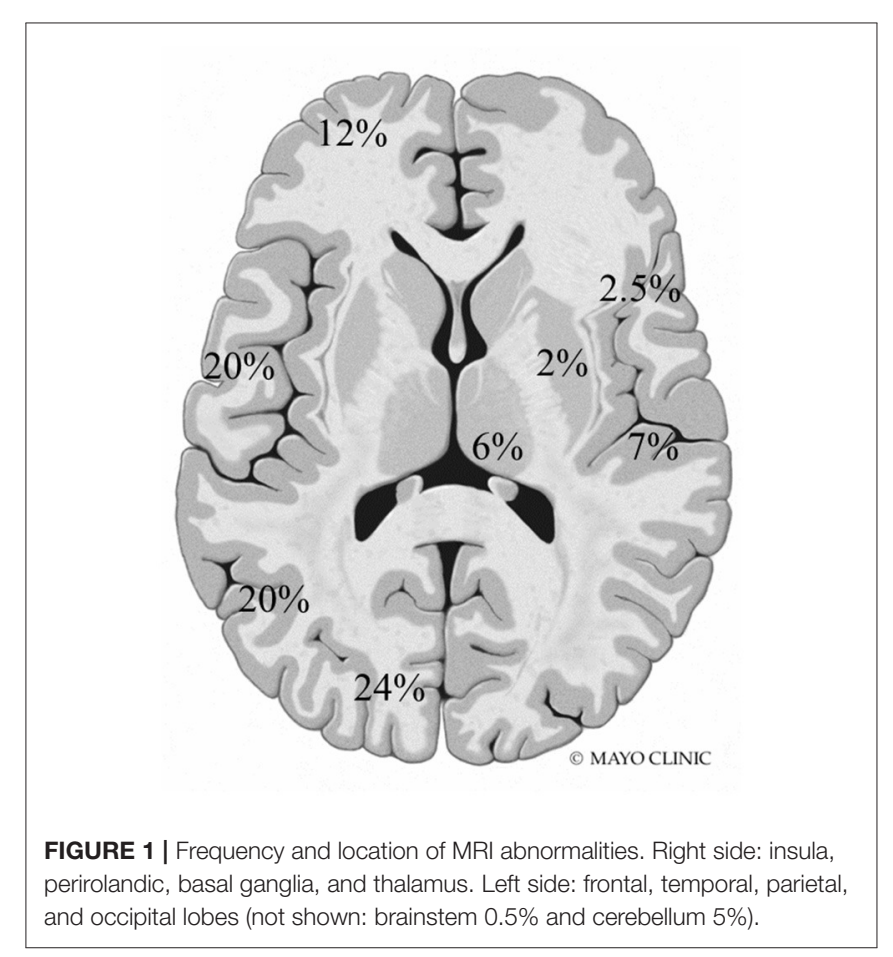

Consecutive MRIs were performed in 17 patients and imaging evidence of disease progression was present in 11 patients [six patients with MELAS, two patients with POLG1, and the patients with MERRF, exosome component 3 (EXOSC3), and dynamin-like protein 1 (DNM1L)]. In the patients with MELAS, T2 hyperintensities and/or DWI abnormalities 

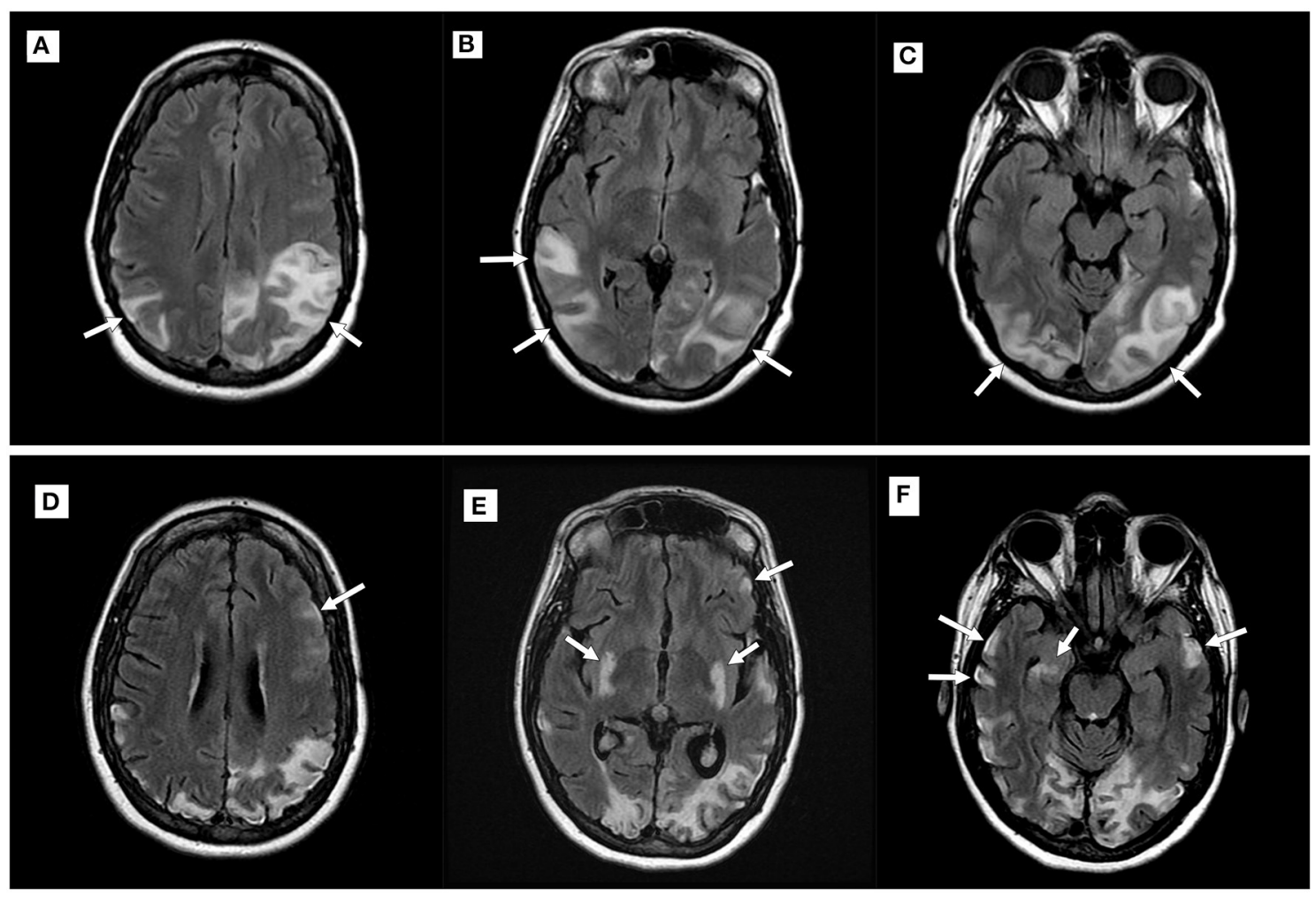

FIGURE 2 | Imaging progression in a patient with mitochondrial encephalomyopathy, lactic acidosis, and stroke-like episodes (MELAS). Top row (A-C): Axial T2 fluid-attenuated inversion recovery (FLAIR) imaging demonstrating new hyperintensities (white arrows) within the parietal and occipital lobes during an acute attack; Bottom row (D-F): Imaging performed during a subsequent MELAS episode with new putaminal and bilateral temporal hyperintensities (white arrows). Note volume loss seen in previously affected areas.

became more widespread over serial examinations, with subsequent abnormalities seen in the bilateral temporoparietal and parieto-occipital regions (Figure 2). In the two patients with POLG1-related disorders, the abnormalities spread to the temporoparietal regions over time. In the patient with MERRF, imaging abnormalities worsened but remained present over the frontal regions. In the patients with EXOCS3 and DNM1L, imaging abnormalities were seen in the parietooccipital regions.

A total of 16 patients had MRI and EEG studies obtained within 30 days of each other (range 0-27 days, mean 7.22 days). A total of 24 MRI-EEG time points were available for review, of which, 13 time points (in nine patients) were concordant and 11 time points (in seven patients) were discordant (Figure 3). The 13 concordant time points corresponded to a period of new or worsening symptoms (i.e., new deficits, new stroke-like attack, breakthrough seizures, status epilepticus, etc.) with 10 of these studies (77\%) performed in the acute inpatient setting. In nine of the discordant time points (82\%), evaluations were performed in the outpatient setting during symptom/disease stability and the remaining two discordant time points occurred during acute episodes of focal motor status epilepticus.

\section{Antiseizure Medications}

A total of 18 patients (90\%) were initiated on ASM therapy during or prior to their first evaluation at our institution. The most commonly prescribed ASMs were levetiracetam $(n=13)$, phenytoin $(n=10)$, lamotrigine $(n=7)$, valproic acid $(n$ $=6)$, and clonazepam $(n=3)$. One patient had a history of hyperammonemia and three patients had a history of transaminitis attributed to valproic acid. The patients on valproic acid were converted to another therapy once a diagnosis of a mitochondrial disorder was made. Side effects occurred in 11 patients (55\%), most commonly reported as mood change or dizziness.

At last known follow-up, the median number of ASMs used were two per patient (range $0-5, \mathrm{SD} 1.5$ ). Of the medications at last follow-up, patients were often on levetiracetam $(n=9)$ and/or lamotrigine $(n=6)$. Five patients experienced at least 1 year of seizure freedom on monotherapy: three patients on lamotrigine, one patient with levetiracetam, and one patient with carbamazepine. One patient had significant reduction on oxcarbazepine to approximately three seizures per year. The remaining four patients on ASM and alive at last follow-up, continued to have refractory epilepsy despite use of one to five ASMs. 

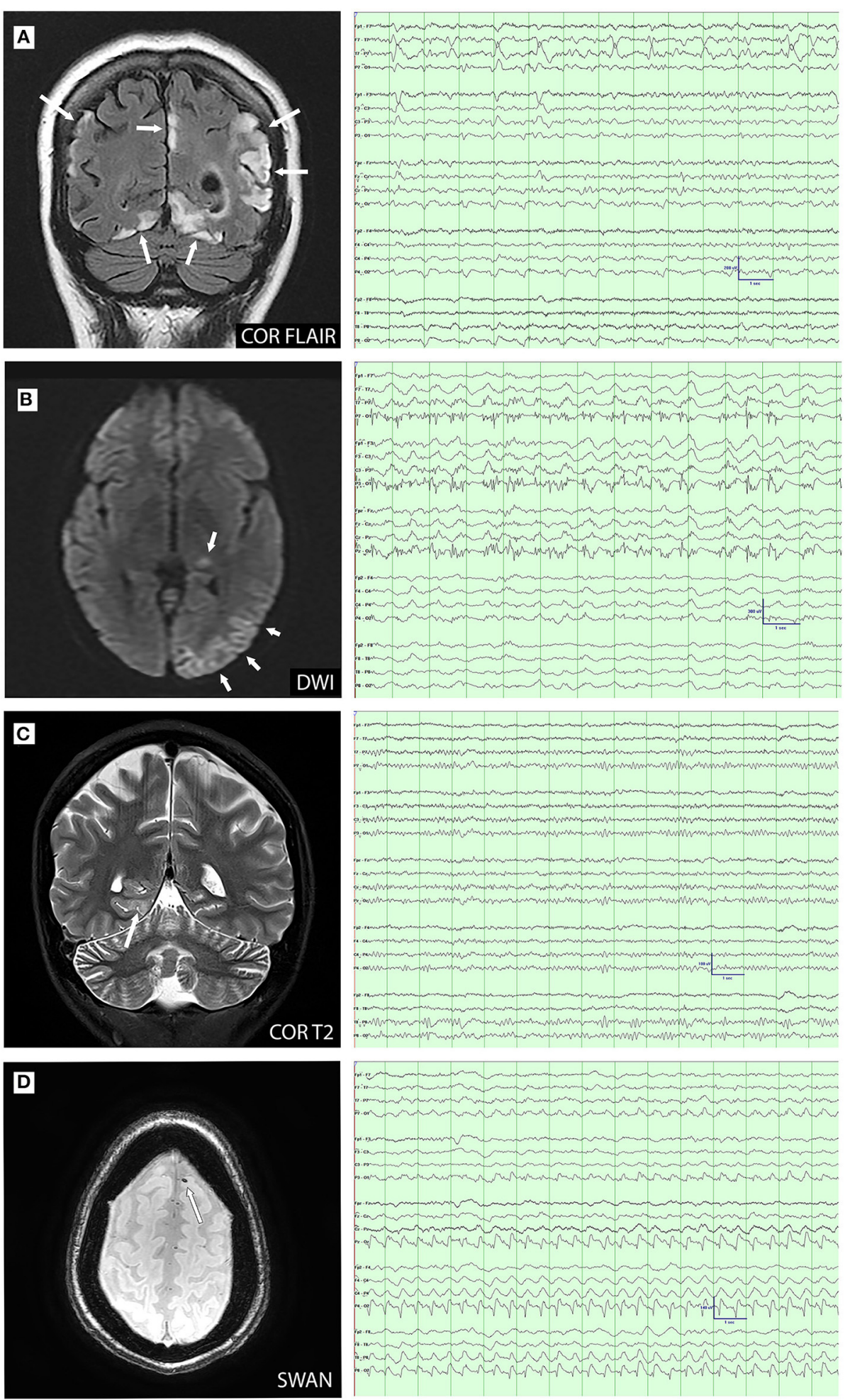

FIGURE 3 | MRI-electroencephalogram (EEG) concordance (A-C) and discordance (D) comparisons. (A): Coronal FLAIR image demonstrates cortical and subcortical hyperintensity in the parietal and occipital lobes. EEG demonstrates left temporal lateral periodic discharges and bioccipital spikes; (B): Diffusion-weighted imaging (DWI) demonstrates restriction in the left pulvinar and temporo-occipital cortex. EEG shows ongoing electrographic seizure activity over the left posterior temporal and occipital; (C): Coronal T2 image demonstrates cortical hyperintensity in the right medial occipitotemporal gyrus. EEG shows right posterior temporal slowing with increased right posterior region amplitudes; and (D): Axial susceptibility-weighted angiography (SWAN) image demonstrates susceptibility artifact consistent with microhemorrhage in the left superior frontal gyrus. EEG demonstrates right posterior lateralized periodic discharges. 
TABLE 3 | Review of selected studies on epilepsy in patients with mitochondrial disorders.

\begin{tabular}{|c|c|c|c|c|c|}
\hline References & $\begin{array}{l}\text { Population (N), Study } \\
\text { type }\end{array}$ & $\begin{array}{l}\text { Disorder(s), genetic } \\
\text { variants }(n)\end{array}$ & Seizure semiology, n (\%) & $\begin{array}{l}\text { EEG epileptiform } \\
\text { abnormality locations } \\
\text { and frequency }\end{array}$ & $\begin{array}{l}\text { Imaging findings location } \\
\text { and frequency }\end{array}$ \\
\hline Fine et al. (27) & $\begin{array}{l}\text { pEDIATRIC and adult (20) } \\
\text { Retrospective }\end{array}$ & $\begin{array}{l}\text { Multiple: } \\
\text { MELAS, } \\
\text { - m.3243A>G (10) } \\
\text { - m.13513G >A (1) } \\
\text { - m.3251A>G (1) } \\
\text { POLG1 } \\
\text {-c.1491G>C, c.2243G >C } \\
\text { (3) } \\
\text { MERRF, m.3256C >T (1) } \\
\text { Leigh, m.13513G>A (1) } \\
\text { PCH type 1B, c.395A>C (1) } \\
\text { DNM1L, c.1207C>T (1) }\end{array}$ & $\begin{array}{l}\text { Focal only } 18(90 \%) \\
\text { Focal to bilateral } \\
\text { tonic-clonic } 6(30 \%) \\
\text { Focal and generalized } 1 \\
(5 \%) \\
\text { Myoclonic } 4(25 \%) \\
\text { Epileptic spasms } 1(5 \%) \\
\text { Status epilepticus } 15(75 \%) \\
\text { - Focal sensory status } \\
7(46.7 \%)\end{array}$ & $\begin{array}{l}\text { Occipital } 40 \% \\
\text { Focal } 55 \% \\
\text { Multifocal } 15 \% \\
\text { Generalized } 25 \% \\
\text { LPDs } 45 \%\end{array}$ & $\begin{array}{l}\text { Occipital/posterior } 44 \% \\
\text { Temporal } 20 \% \\
\text { Frontal } 12 \% \\
\text { Thalamus 6\% } \\
\text { Basal ganglia } 2 \% \\
\text { Global atrophy } 80 \%\end{array}$ \\
\hline Li et al. (28) & $\begin{array}{l}\text { Pediatric and adult (34) } \\
\text { Retrospective }\end{array}$ & MELAS, m.3243A>G (34) & $\begin{array}{l}\text { Focal } 20 \text { (58.8\%) } \\
\text { Generalized } 11(32.3 \%) \\
\text { Unknown } 3(8.8 \%)\end{array}$ & $\begin{array}{l}\text { Focal } 69.57 \% \\
\text { Occipital } 69.57 \% \\
\text { Frontal } 65.22 \% \\
\text { Temporal } 47.83 \% \\
\text { Parietal } 34.78 \%\end{array}$ & $\begin{array}{l}\text { Occipital } 75 \% \\
\text { Temporal } 85.71 \% \\
\text { Parietal } 85.71 \% \\
\text { Atrophy } 64.29 \%\end{array}$ \\
\hline Lee et al. (8) & $\begin{array}{l}\text { Pediatric (22) } \\
\text { Retrospective }\end{array}$ & MELAS, m.3243A>G (22) & $\begin{array}{l}\text { Focal only (50\%), } \\
\text { Focal and generalized } \\
\text { (13.6\%) } \\
\text { Generalized only (1\%) } \\
\text { Status epilepticus (100\%) }\end{array}$ & $\begin{array}{l}\text { Focal } 36.4 \% \\
\text { Multifocal } 31.2 \% \\
\text { Generalized } 27.3 \% \\
\text { LPDs } 31.8 \%\end{array}$ & $\begin{array}{l}\text { Infarction } 90.9 \% \text { (location } \\
\text { not specified) } \\
\text { Basal ganglia } 50 \% \\
\text { Thalamus } 8 \% \\
\text { White matter } 45.5 \% \\
\text { Atrophy } 77.3 \%\end{array}$ \\
\hline $\begin{array}{l}\text { Demarest et al. } \\
\text { (29) }\end{array}$ & $\begin{array}{l}\text { Pediatric (7) } \\
\text { Retrospective }\end{array}$ & MELAS, m.3243A> G (7) & $\begin{array}{l}\text { Focal } 5 \text { (71\%) } \\
\text { Status epilepticus } 4 \text { (57\%) } \\
\text { - Occipital status } \\
\text { epilepticus } 2 \text { (50\%) }\end{array}$ & $\begin{array}{l}\text { Focal } 57 \% \\
\text { Generalized } 14 \% \\
\text { Occipital/posterior } 37.5 \% \\
\text { Frontal } 12.5 \% \\
\text { LPDs } 12.5 \%\end{array}$ & $\mathrm{~N} / \mathrm{A}$ \\
\hline $\begin{array}{l}\text { Specchio et al. } \\
\text { (16) }\end{array}$ & $\begin{array}{l}\text { Pediatric and adult (195) } \\
\text { Meta-analysis/Review }\end{array}$ & $\begin{array}{l}\text { POLG } \\
- \text { c. } 1399 G>A \\
-c .2243 G>C \\
-c .2542 G>A \\
\text { (74.2\% of variants identified) }\end{array}$ & $\begin{array}{l}N=183 \\
\text { Myoclonic } 95(52 \%) \\
\text { Focal motor } 62(34 \%) \\
\text { Focal to bilateral } \\
\text { tonic-clonic } 81(44 \%) \\
\text { Status epilepticus } 85(46 \%)\end{array}$ & $\begin{array}{l}N=77 \\
\text { Occipital } 19.5 \% \\
\text { Centroparietal 14\% } \\
\text { Diffuse } 6.5 \% \\
\text { LPDs } 1 \%\end{array}$ & $\begin{array}{l}N=109 \\
\text { Occipital 38\% } \\
\text { Temporal/parietal 15.5\% } \\
\text { Frontal 9\% } \\
\text { Thalamus } 40 \% \\
\text { Basal ganglia } 5.5 \% \\
\text { Atrophy } 26.6 \%\end{array}$ \\
\hline $\begin{array}{l}\text { Anagnostou et al. } \\
\text { (10) }\end{array}$ & $\begin{array}{l}\text { Pediatric and adult (372) } \\
\text { Meta-analysis/Review }\end{array}$ & $\begin{array}{l}\text { POLG } \\
\text { - c.1399G>A (215) } \\
\text { - c.2243G>C (70) } \\
\text { - c. } 2542 G>A(26)\end{array}$ & $\begin{array}{l}N=229 \text { Focal motor } \\
146(64 \%) \text { Myoclonic } \\
133(58 \%) \text { Status } \\
\text { epilepticus - Generalized } \\
\text { status } 112(49 \%) \text { - Focal } \\
\text { motor status } 78(34 \%)\end{array}$ & $\begin{array}{l}N=72 \\
\text { Occipital/posterior } 61 \% \\
\text { Frontal } 6 \% \\
\text { Temporal } 2 \% \\
\text { Multifocal 23\% }\end{array}$ & $\begin{array}{l}N=136 \text { Stroke-like } \\
\text { lesion 43\% -Occipital 86\% } \\
\text {-Parietal 20\% -Frontal 7\% } \\
\text {-Temporal 1.5\% Thalamus } \\
\text { 37\% Basal ganglia 14\% } \\
\text { Atrophy 28\% }\end{array}$ \\
\hline $\begin{array}{l}\text { Chevallier et al. } \\
(30)\end{array}$ & $\begin{array}{l}\text { Pediatric and adult (165) } \\
\text { Retrospective }\end{array}$ & $\begin{array}{l}\text { Multiple (detailed genetic } \\
\text { variants not available): } \\
\text { Leigh (16) } \\
\text { MELAS (9) } \\
\text { POLG/SANDO (2) } \\
\text { MERRF } \\
\text { DNA depletion syndrome (5) }\end{array}$ & $\begin{array}{l}N=60 \\
\text { Focal } 43 \% \\
\text { Generalized tonic-clonic } \\
37 \% \\
\text { Myoclonic } 22 \% \\
\text { Infantile spasms } 7 \%\end{array}$ & $\begin{array}{l}N=109 \\
\text { Focal 39\% } \\
\text { Generalized 39\%, } \\
\text { Multifocal 41\% } \\
\text { Generalized and } \\
\text { multifocal 25\% }\end{array}$ & $\mathrm{N} / \mathrm{A}$ \\
\hline $\begin{array}{l}\text { Lorenzoni et al. } \\
\text { (31) }\end{array}$ & $\begin{array}{l}\text { Adult (6) } \\
\text { Retrospective }\end{array}$ & $\begin{array}{l}\text { MERRF } \\
-m .8344 A>G(5)\end{array}$ & $\begin{array}{l}\text { Myoclonic } 6(100 \%) \\
\text { Generalized tonic-clonic } \\
6(100 \%)\end{array}$ & Generalized 83\% & Diffuse atrophy 66\% \\
\hline
\end{tabular}

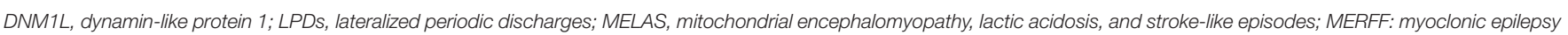
with ragged-red fibers; $P C H$, pontocerebellar hypoplasia; POLG, polymerase gamma; SANDO, sensory ataxia, neuropathy, dysarthria, and ophthalmoparesis.

\section{Nutraceutical Supplementation (Mitochondrial Cocktail)}

All patients were initiated on nutraceutical supplementation at the time of diagnosis and all patients remained on supplementation at last follow-up or time of death. The most commonly used supplements were carnitine (95\%), coenzyme Q10 (90\%), arginine (40\%), and riboflavin (40\%) with the number of supplements at initial evaluation ranging from one to 
nine (mean 3.9, SD 1.9). There was no significant difference seen between number of supplements used and clinical progression. In a subanalysis of 10 patients with MELAS, there was no statistically significant difference between the mean number of supplements and subsequent stroke-like episodes $(p=0.7)$. In adult patients who were still living at the end of the study period, there was no relationship seen between use of supplements and seizure control ( $p=0.39)$, level of independence $(p=0.27)$, or mortality $(p=0.13)$.

\section{Functional Status and Follow-Up}

The duration of follow-up ranged from 3 months to 31 years (mean 9 years). At last follow-up or patient contact, eight patients (40\%) were deceased [five patients with MELAS (one patient with Ogilvie's syndrome/toxic megacolon, one patient with cancer, one patient with respiratory arrest, and two patients related to disease progression)], one patient with POLG (withdrawal of support in refractory myoclonic status epilepticus), one patient with Leigh syndrome (respiratory arrest), and one patient with DNM1L (withdrawal of support in refractory myoclonic status epilepticus). All the patients were assigned an initial and followup mRS score (Table 1). The mean initial mRS was 1 (range 04 , SD 1.2), correlating to no significant disability, compared to last mRS mean of 4.65 (range 2-6, SD 1.4), corresponding to a moderately severe to severe disability and requiring assistance for all the needs. At last follow-up, no patients were $\mathrm{mRS}=0$.

\section{DISCUSSION}

This retrospective series of patients with mitochondrial disorders and epilepsy demonstrated an increasing predominance of posterior imaging and EEG abnormalities. Focal seizures, particularly with visual phenomena, were the most frequently seen, correlating with focal lesions commonly found in our cohort. As expected, patients with the same genetic variant had variable disease manifestations and clinical course.

On EEG studies, seizures and epileptiform abnormalities most commonly localized to the posterior temporal, parietal, and occipital regions. This proclivity for the posterior cerebral regions in mitochondrial disorders has been previously described and early recognition of such findings should suggest the possibility of a mitochondrial cytopathy $(7,15,26)$.

Our findings are supported by previous patient series and literature reviews on epilepsy features in mitochondrial disorders (Table 3) $(8,10,16,28-31)$. Focal seizures were the predominant seizure type in our series, which is in agreement with the previous review by Anagnostou et al. in POLG and a recent case series in MELAS by $\mathrm{Li}$ et al. $(10,28)$. In a series of 19 patients with POLG1, occipital rhythmic slowing was present in 7 patients and occipital interictal discharges was present in 18 patients, demonstrating an occipital predilection in POLG (15). This finding, as seen in our study over the disease course, is concordant with electrophysiologic data reported by other authors $(10,16,28,29)$. Occipital status epilepticus and occipital seizures have also been previously reported in MELAS; this manifestation may precede other clinical signs of the disease and serve as a potential diagnostic clue $(29,32,33)$. Similarly, in our series, MRI abnormalities frequently involved the posterior head regions, often in concordance with EEG findings, which is in agreement with other series $(18,34,35)$.

The most common mitochondrial disorder in our cohort was MELAS. Clinically, this diagnosis should be considered in an individual, particularly a young individual, with recurrent stroke-like episodes of unclear etiology. Stroke in MELAS is hypothesized to result from a combination of impaired oxidative phosphorylation, abnormal nitric oxide homeostasis, and vascular endothelial dysfunction (36-38). Oxidative stress leads to abnormal calcium homeostasis producing neuronal hyperexcitability $(39,40)$. The resultant localized cortical dysfunction and cerebral edema in acute and recovering cerebral infarcts increases the risk for seizure. Seizures then lead to increased free radical production within dysfunctional mitochondria, predisposing to further oxidative damage (5). With prolonged seizures, this process may lead to cortical laminar necrosis, which is manifested in the acute phase as T2 hyperintense or DWI hyperperfusion signal in the cortical ribbon on MRI $(37,41)$. The combination of local cortical injury and ongoing seizures then could produce a structural lesion (i.e., encephalomalacia, gliosis, etc.) predisposing to future seizure activity.

Inexorable progression was seen in our patients, with recurrent seizures, stroke-like episodes, and further brain injury, leading to continued functional decline. The majority developed moderate-to-severe disability or death, with eight patients deceased, of which seven deaths were attributable to their underlying mitochondrial disorder.

The treatment of seizures in patients with mitochondrial disorders is challenging. There is known mitochondrial toxicity from several ASMs, notably, valproic acid, phenytoin, and phenobarbital $(42,43)$. In our series, it was rare for patients to not have tried two or more ASMs including valproic acid, phenytoin, and phenobarbital. Interestingly, two patients were not started on ASM while seen at our institution, despite recurrent seizures. The reason documented for both was patient/family preference. The clinical course of the epilepsy in this cohort was unfortunately dismal, with the majority experiencing intractability and two of whom died from complications of superrefractory status epilepticus.

We found no evidence of efficacy of nutraceutical supplementation in our cohort, with the majority experiencing significant progression and exacerbations despite treatment. Compliance with the mitochondrial cocktail was difficult to assess based on chart review alone, which limited our ability to be certain as to the futility of therapy. These regimens can be difficult to maintain and are cost prohibitive. Some authors report the benefits of arginine and citrulline during acute attacks in MELAS to mitigate cortical injury by free radical reduction $(36,44,45)$. Indeed, our practice is to include arginine in the regimen of patients with MELAS and provide additional supplementation during a stroke-like episode. However, the results of this retrospective review are sobering as to the potential for response to nutritional therapies in these patients. The use of other supplements has not been shown to alter disease course in mitochondrial disorders (45). A prospective clinical trial would 
be necessary to definitively evaluate the efficacy of supplement use in mitochondrial disorders.

There are several limitations of this study. As this study was retrospective, we were limited to information contained within the medical record. A large number of patients were excluded due to missing or incomplete records. We only included those with genetically confirmed disease, thus further reducing our sample size and potentially limiting our cohort to the most severely affected. Our small series was heavily weighted toward patients with MELAS, which could have skewed our findings and interpretation.

\section{CONCLUSION}

This retrospective series reviewed the clinical course of 20 patients with mitochondrial disorders and epilepsy. Most patients had EEG and neuroimaging abnormalities over the posterior head regions or showed progression to involvement of these regions over time. Seizures with visual symptoms occurred frequently. The presence of posterior predominant EEG and MRI abnormalities of unclear etiology should increase clinical suspicion of a mitochondrial disorder, particularly in an adult patient presenting with occipital status epilepticus. Our results raise question as to the efficacy of nutraceutical therapy for these diseases. A larger prospective study would be helpful to

\section{REFERENCES}

1. Poole OV, Hanna MG, Pitceathly RD. Mitochondrial disorders: disease mechanisms and therapeutic approaches. Discov Med. (2015) 20:325-31. Available online at: https://www.discoverymedicine.com/Olivia-V-Poole/ 2015/11/mitochondrial-disorders-disease-mechanisms-and-therapeuticapproaches/

2. Zeviani M. Mitochondrial disorders. Suppl Clin Neurophysiol. (2004) 57:30412. doi: 10.1016/S1567-424X(09)70366-6

3. DiMauro S. Mitochondrial diseases. Biochim Biophys Acta. (2004) 1658:80-8. doi: 10.1016/j.bbabio.2004.03.014

4. Haas RH, Zolkipli Z. Mitochondrial disorders affecting the nervous system. Semin Neurol. (2014) 34:321-40. doi: 10.1055/s-0034-1386770

5. Patel M. Mitochondrial dysfunction and oxidative stress: cause and consequence of epileptic seizures. Free Radic Biol Med. (2004) 37:1951-62. doi: 10.1016/j.freeradbiomed.2004.08.021

6. Uusimaa J, Gowda V, McShane A, Smith C, Evans J, Shrier A, et al. Prospective study of POLG mutations presenting in children with intractable epilepsy: prevalence and clinical features. Epilepsia. (2013) 54:1002-11. doi: $10.1111 /$ epi.12115

7. Hikmat $\mathrm{O}$, Eichele T, Tzoulis C, Bindoff LA. Understanding the epilepsy in POLG related disease. Int J Mol Sci. (2017) 18:1845. doi: $10.3390 / \mathrm{ijms} 18091845$

8. Lee HN, Eom S, Kim SH, Kang HC, Lee JS, Kim HD, et al. Epilepsy characteristics and clinical outcome in patients with mitochondrial encephalomyopathy, lactic acidosis, and stroke-like episodes (MELAS). Pediatr Neurol. (2016) 64:59-65. doi: 10.1016/j.pediatrneurol.2016.08.016

9. Finsterer J, Zarrouk-Mahjoub S. Management of epilepsy in MERRF syndrome. Seizure. (2017) 50:166-70. doi: 10.1016/j.seizure.2017.06.010

10. Anagnostou ME, Ng YS, Taylor RW, McFarland R. Epilepsy due to mutations in the mitochondrial polymerase gamma (POLG) gene: A clinical and molecular genetic review. Epilepsia. (2016) 57:1531-45. doi: $10.1111 /$ epi.13508

11. Finsterer J, Zarrouk Mahjoub S. Mitochondrial epilepsy in pediatric and adult patients. Acta Neurol Scand. (2013) 128:141-52. doi: 10.1111/ane.12122 further clarify the optimal medical management of patients with mitochondrial disorders and epilepsy.

\section{DATA AVAILABILITY STATEMENT}

The original contributions presented in the study are included in the article/supplementary material, further inquiries can be directed to the corresponding author/s.

\section{ETHICS STATEMENT}

The studies involving human participants were reviewed and approved by Mayo Clinic IRB. Written informed consent from the participants' legal guardian/next of kin was not required to participate in this study in accordance with the national legislation and the institutional requirements.

\section{AUTHOR CONTRIBUTIONS}

AF wrote the first draft of the manuscript, collected, analyzed and interpreted data, and revised the manuscript. GL collected and interpreted data and revised the manuscript. RG and JB revised the manuscript and gave final approval. All the authors contributed to the article and approved the submitted version.
12. Finsterer J, Zarrouk Mahjoub S. Epilepsy in mitochondrial disorders. Seizure. (2012) 21:316-21. doi: 10.1016/j.seizure.2012.03.003

13. Canafoglia L, Franceschetti S, Antozzi C, Carrara F, Farina L, Granata T, et al. Epileptic phenotypes associated with mitochondrial disorders. Neurology. (2001) 56:1340-6. doi: 10.1212/WNL.56.10.1340

14. Serra G, Piccinnu R, Tondi M, Muntoni F, Zeviani M, Mastropaolo C. Clinical and EEG findings in eleven patients affected by mitochondrial encephalomyopathy with MERRF-MELAS overlap. Brain Dev. (1996) 18:18591. doi: 10.1016/0387-7604(95)00147-6

15. Engelsen BA, Tzoulis C, Karlsen B, Lillebo A, Laegreid LM, Aasly J, et al. POLG1 mutations cause a syndromic epilepsy with occipital lobe predilection. Brain. (2008) 131:818-28. doi: 10.1093/brain/awn007

16. Specchio N, Pietrafusa N, Calabrese C, Trivisano M, Pepi C, de Palma L, et al. POLG1-related epilepsy: review of diagnostic and therapeutic findings. Brain Sci. (2020) 10:10768. doi: 10.3390/brainsci10110768

17. Alves C, Goncalves FG, Grieb D, Lucato LT, Goldstein AC, Zuccoli G. Neuroimaging of mitochondrial cytopathies. Top Magn Reson Imaging. (2018) 27:219-40. doi: 10.1097/RMR.0000000000000173

18. Mascalchi M, Montomoli M, Guerrini R. Neuroimaging in mitochondrial disorders. Essays Biochem. (2018) 62:409-21. doi: 10.1042/EBC20170109

19. Tarnopolsky MA. The mitochondrial cocktail: rationale for combined nutraceutical therapy in mitochondrial cytopathies. Adv Drug Deliv Rev. (2008) 60:1561-7. doi: 10.1016/j.addr.2008.05.001

20. Bernier FP, Boneh A, Dennett X, Chow CW, Cleary MA, Thorburn DR. Diagnostic criteria for respiratory chain disorders in adults and children. Neurology. (2002) 59:1406-11. doi: 10.1212/01.WNL.0000033795.17156.00

21. Walker UA, Collins S, Byrne E. Respiratory chain encephalomyopathies: a diagnostic classification. Eur Neurol. (1996) 36:260-7. doi: 10.1159/000117269

22. Morava E, van den Heuvel L, Hol F, de Vries MC, Hogeveen M, Rodenburg RJ, et al. Mitochondrial disease criteria: diagnostic applications in children. Neurology. (2006) 67:1823-6. doi: 10.1212/01.wnl.0000244435.2 7645.54

23. Wolf NI, Smeitink JA. Mitochondrial disorders: a proposal for consensus diagnostic criteria in infants and children. Neurology. (2002) 59:1402-5. doi: 10.1212/01.WNL.0000031795.91814.D8 
24. Fisher RS, Cross JH, D'Souza C, French JA, Haut SR, Higurashi N, et al. Instruction manual for the ILAE 2017 operational classification of seizure types. Epilepsia. (2017) 58:531-42. doi: 10.1111/epi.13671

25. Scheffer IE, Berkovic S, Capovilla G, Connolly MB, French J, Guilhoto L, et al. ILAE classification of the epilepsies: Position paper of the ILAE Commission for Classification and Terminology. Epilepsia. (2017) 58:512-21. doi: 10.1111/epi.13709

26. Khurana DS, Valencia I, Goldenthal MJ, Legido A. Mitochondrial dysfunction in epilepsy. Semin Pediatr Neurol. (2013) 20:176-87. doi: 10.1016/j.spen.2013.10.001

27. Fine AL, Liebo G, Gavrilova RH, Britton JW. Seizure semiology, EEG, and imaging findings in epilepsy secondary to mitochondrial disease. Front. Neurol. (2021). 12:779052. doi: 10.3389/fneur.2021.779052

28. Li J, Zhang W, Cui Z, Li Z, Jiang T, Meng H. Epilepsy associated with mitochondrial encephalomyopathy, lactic acidosis, and stroke-like episodes. Front Neurol. (2021) 12:675816. doi: 10.3389/fneur.2021.675816

29. Demarest ST, Whitehead MT, Turnacioglu S, Pearl PL, Gropman AL. Phenotypic analysis of epilepsy in the mitochondrial encephalomyopathy, lactic acidosis, and strokelike episodes-associated mitochondrial DNA A3243G mutation. J Child Neurol. (2014) 29:1249-56. doi: 10.1177/0883073814538511

30. Chevallier JA, Von Allmen GK, Koenig MK. Seizure semiology and EEG findings in mitochondrial diseases. Epilepsia. (2014) 55:707-12. doi: 10.1111/epi.12570

31. Lorenzoni PJ, Scola RH, Kay CS, Silvado CE, Werneck LC. When should MERRF (myoclonus epilepsy associated with ragged-red fibers) be the diagnosis? Arq Neuropsiquiatr. (2014) 72:803-11. doi: 10.1590/0004-282X20140124

32. Cesaroni E, Scarpelli M, Zamponi N, Polonara G, Zeviani M. Mitochondrial encephalomyopathy lactic acidosis and strokelike episodes mimicking occipital idiopathic epilepsy. Pediatr Neurol. (2009) 41:131-4. doi: 10.1016/j.pediatrneurol.2009.02.018

33. Karkare S, Merchant S, Solomon G, Engel M, Kosofsky B. MELAS with A3243G mutation presenting with occipital status epilepticus. J Child Neurol. (2009) 24:1564-7. doi: 10.1177/0883073809334386

34. Finsterer J. Features on cerebral imaging suggesting mitochondrial disorder. Neurodegener Dis Manag. (2018) 8:215-6. doi: 10.2217/nmt-2018-0015

35. Bindu PS, Arvinda H, Taly AB, Govindaraju C, Sonam K, Chiplunkar S, et al. Magnetic resonance imaging correlates of genetically characterized patients with mitochondrial disorders: A study from south India. Mitochondrion. (2015) 25:6-16. doi: 10.1016/j.mito.2015.08.002

36. Koga Y, Povalko N, Nishioka J, Katayama K, Kakimoto N, Matsuishi T. MELAS and L-arginine therapy: pathophysiology of stroke-like episodes. Ann N Y Acad Sci. (2010) 1201:104-10. doi: 10.1111/j.1749-6632.2010.05624.x
37. Ito H, Mori K, Harada M, Minato M, Naito E, Takeuchi M, et al. Serial brain imaging analysis of stroke-like episodes in MELAS. Brain Dev. (2008) 30:483-8. doi: 10.1016/j.braindev.2008.01.003

38. Iizuka T, Sakai F, Kan S, Suzuki N. Slowly progressive spread of the stroke-like lesions in MELAS. Neurology. (2003) 61:1238-44. doi: 10.1212/01.WNL.0000091888.26232.FE

39. Kunz WS. The role of mitochondria in epileptogenesis. Curr Opin Neurol. (2002) 15:179-84. doi: 10.1097/00019052-200204000-00009

40. Iizuka T, Sakai F, Suzuki N, Hata T, Tsukahara S, Fukuda M, et al. Neuronal hyperexcitability in stroke-like episodes of MELAS syndrome. Neurology. (2002) 59:816-24. doi: 10.1212/WNL.59.6.816

41. Valanne L, Paetau A, Suomalainen A, Ketonen L, Pihko H. Laminar cortical necrosis in MELAS syndrome: MR and neuropathological observations. Neuropediatrics. (1996) 27:154-60. doi: 10.1055/s-2007-973767

42. Finsterer J. Toxicity of antiepileptic drugs to mitochondria. Handb Exp Pharmacol. (2017) 240:473-88. doi: 10.1007/164_2016_2

43. Finsterer J, Scorza FA. Effects of antiepileptic drugs on mitochondrial functions, morphology, kinetics, biogenesis, and survival. Epilepsy Res. (2017) 136:5-11. doi: 10.1016/j.eplepsyres.2017.07.003

44. El-Hattab AW, Emrick LT, Chanprasert S, Craigen WJ, Scaglia F. Mitochondria: role of citrulline and arginine supplementation in MELAS syndrome. Int J Biochem Cell Biol. (2014) 48:85-91. doi: 10.1016/j.biocel.2013.12.009

45. Ganetzky RD, Falk MJ. 8-year retrospective analysis of intravenous arginine therapy for acute metabolic strokes in pediatric mitochondrial disease. $\mathrm{Mol}$ Genet Metab. (2018) 123:301-8. doi: 10.1016/j.ymgme.2018.01.010

Conflict of Interest: The authors declare that the research was conducted in the absence of any commercial or financial relationships that could be construed as a potential conflict of interest.

Publisher's Note: All claims expressed in this article are solely those of the authors and do not necessarily represent those of their affiliated organizations, or those of the publisher, the editors and the reviewers. Any product that may be evaluated in this article, or claim that may be made by its manufacturer, is not guaranteed or endorsed by the publisher.

Copyright (C) 2021 Fine, Liebo, Gavrilova and Britton. This is an open-access article distributed under the terms of the Creative Commons Attribution License (CC BY). The use, distribution or reproduction in other forums is permitted, provided the original author(s) and the copyright owner(s) are credited and that the original publication in this journal is cited, in accordance with accepted academic practice. No use, distribution or reproduction is permitted which does not comply with these terms. 